\title{
Visualization of the sustainable development model of the green core of the agglomeration: the neogeography of the Chelyabinsk urban pine forest
}

\author{
Sergey Gordeev ${ }^{1, *}$, Andrey Kocherov ${ }^{2}$, and Vera Merker $^{3}$ \\ ${ }^{1}$ Chelyabinsk branch of Russian Presidential Academy of National Economy and Public \\ Administration, 26, Komarova st., 454077, Chelyabinsk, Russia \\ ${ }^{2}$ South Ural State University (National Research University), 76, Lenin Prospect, 454080, \\ Chelyabinsk, Russia \\ ${ }^{3}$ Chelyabinsk State University, 129, Br. Kashirinykh st., 454001, Chelyabinsk, Russia
}

\begin{abstract}
In the study on building a model of spatial development, the issues of problem-oriented zoning of territories, transformation of the settlement framework, integration of the most significant objects of social transformations are considered. Such objects in the heterogeneous social Eurasian space are, first of all, the zones of agglomerations. Taking into account the regional specifics when identifying a heterogeneous set of key spatial objects, the main provisions of the spatial development of the territory as a complex multi-level socio-economic system are determined. The solution to the issues of strategic planning of such systems is associated with the problematic adaptation of visualization technologies, the joint use of a special format of cartographic and digital information. The main research results create the prerequisites for a significant expansion of the number of tasks of managing regional and municipal development of the natural and ecological framework.
\end{abstract}

\section{Introduction}

Global structural changes in the economy, transformations of social space, and an increase in the load on the environment are increasingly manifested at the level of various spatial systems, including natural systems. Designing the spatial development of urban systems in such conditions is significantly difficult. The historically inherited trajectory of spatial transformation is changing due to environmental, social and economic consequences. These changes contribute to the rapid obsolescence of master plans for the development of cities and territorial schemes.

The ineffectiveness of spatial development is especially noticeable when the natural environment is significantly disturbed. Special problems are typical for territories where the degree of anthropogenic load (the magnitude of the direct or indirect impact of people and their economic activity on the components of natural systems) is many times higher than the

\footnotetext{
*Corresponding author: sgordeev222@gmail.com
} 
permissible level. Many different problems associated with a significant increase in anthropogenic load are observed in most industrially developed territories. A balanced change in the main parameters of the development of systems will reduce the risks of conflict between its structural elements. At the same time, the best socio-economic results are provided and the negative consequences of technogenesis (undesirable changes in landscapes under the influence of industrial and recreational human activities) are reduced.

Recently, rational use of natural resources has become especially important, guaranteeing that the exploitation of resources can be carried out indefinitely. Such conditions are usually considered within the framework of the problem of sustainable development. This problem touches upon many categories and interpretations of development at the level of both global and local systems. Sustainable development issues are especially important for heterogeneous spatial systems, where economic, social and environmental factors have complex and specific interrelationships. Recently, more and more sustainable development goals are linked to environmental conservation issues.

Changes in the conditions for preserving the environment and, accordingly, socioeconomic development are most noticeable for some urban spatial systems, including agglomerations. Agglomerations as dynamic rapidly transforming spatial systems with the development of communications integrate many objects of the urban environment associated with high economic growth and environmental stress. Agglomerations can include various natural, including unique, objects and become a driver of regional transformations. But at the same time, in the territories of agglomerations, with a significant development of the economy, the threats of exceeding the permissible anthropogenic impact on the biosphere and the threat of irreversible consequences of anthropogenic impacts on natural objects in the city or elements of the natural-ecological framework increase.

The complex interrelationships of the processes that determine the development of an agglomeration as a complex system impose additional requirements on the construction of models of such transformations. In such conditions, the construction of models for the development of key objects of agglomerations is of particular importance. Among them, the most significant are objects in the field of nature management. In models of sustainable development of large cities with a large number of indicators, environmental management objects are usually considered as homogeneous point objects [1].

Expanding the possibilities of studying the transformations of complex, heterogeneous urban spatial systems (when considering a set of objects) is associated with a qualitative update of information support. In such studies, a special place is occupied by the use of problem-adapted computer graphics - data visualization technology. Using the system pine forest - urban space as an example, the author examines the transformation of a complex, heterogeneous territory in terms of the structure of the territory under conditions of an increase in anthropogenic (recreational) load.

The scale and acuteness of the internal contradictions of such a system makes it a reference object for the study of a number of problems of sustainable development. The key role in the study is acquired by the problematic adaptation of geoinformation technologies associated with the zoning of territories according to various criteria, which significantly expands the possibilities of analyzing the prospects. The methodology for constructing a problem-oriented model opens up opportunities for a productive solution to the problem of system development and the corresponding management decisions.

\section{Materials and Methods}

In the context of multiple changes, it is unrealistic to build a universal model for the transformation of open spatial systems with a degree of detail sufficient for practical use. With the difference even in the main objects of the natural resource framework of the 
agglomeration, the number of factors and development scenarios under consideration increases many times. Added to this are the problems of formalization and lack of information. Such conditions predetermine the procedure for a sequential multistage study of the transformation of an agglomeration as a complex system.

Initially, the factors of transformation of the system are classified with the allocation of key objects, including natural ones (within the framework of the requirements of the sustainable development model). This is followed by the systematization of the most significant regularities of transformation, the determination of the trajectories of possible development of both key natural objects and the spatial system as a whole.

The possibilities for solving many problems of spatial development are associated with new sources and volumes of information about the Earth's surface (data of remote sensing of the Earth using geoinformation systems). New geoinformation data, supplementing existing sources of information, can significantly expand the understanding of the current state and relationships of spatial objects. The primary source of geoinformation data is the data of space and aerial photographs.

They are available in public information systems (geospatial service Google Earth, etc.). Processing of photographs is associated with the preparation of the appropriate toolkit (pattern recognition, Big Data, artificial intelligence, etc.). Such an addition to management technologies is considered as an important component of the new direction of regional development management [2].

The creation of a new geoinformation environment (with an increase of several orders of magnitude in the amount of data) makes it possible to distinguish a new class of mathematical models of regional development - neogeography models [3]. The important properties of this class of models are the use of raster (not vector) representation of geographic information, the integration of different-quality layers of space and the delimitation of zones according to a certain set of social, environmental and economic indicators. These qualities are especially important in this study. The construction of such models has a number of features and is limited by the capabilities of the geospatial service [4].

The considered class of models is directly related to the visualization and aggregation of information of different quality. Among the graphic models that display a territorial object at different levels of abstraction and formalization, such models occupy their definite, intermediate place between classical maps and abstract schemes. The graphical component of such models may differ significantly from conventional and digital geographical maps and geographical information systems (such as geographical cartogram [5]. Such models reflect the development of technologies for using problem-oriented graphic information and form the basis for digital assessments.

Determination of the main parameters of neogeographic models for a specific object, which is a large green zone in the core of the agglomeration, is associated with the adaptation of a number of provisions of sustainable development. Many general provisions of sustainable development at the global level require clarification in the study of local spatial systems. In this case, adaptation is associated with the preparation of a system of restrictions and priorities for the transformation of the forest and the surrounding area (taking into account the many factors that determine the anthropogenic load).

In the initial formulation, the problem of sustainable development is based on the obvious statement - a closed system using limited resources cannot grow indefinitely. The limits to the growth of the system are determined by the volume of these resources and the requirement to meet needs without prejudice to future generations.

Sustainable development is considered to be a unifying moment in the management of economic, environmental and social processes with a detailed system of goals [6]. However, when considering the goals of sustainable development in a real multi-level management system with existing institutions, a number of contradictions inevitably arise. Economic 
development issues are extraterritorial within the framework of the national economy. The management of many social life processes is the responsibility of the regions. Many environmental issues (nature management and environmental protection) are concentrated at the local, municipal level.

In local spatial systems, these capabilities differ significantly. The imbalance of interests for such systems is real. In such a situation, for the system under consideration, based on its specifics, a number of additional clarifying provisions of sustainable development are determined:

- The issues of finding solutions are largely determined by the effectiveness of compromises (the ratio of losses and gains);

- The forecasting period is determined by the parameters of the development of the main object (in our case, the unique natural characteristics of the forest);

- Issues of preserving the environment are considered within the limits of permissible anthropogenic impact on the biosphere (up to the border of irreversible consequences). Further, they act in the format of restrictions (for example: conservation of natural biological diversity is mandatory);

- Around any long-term spatial object of the system under consideration, a relatively stable complex of other objects is inevitably formed, changing its characteristics;

- In the problem of sustainable development, there are many social issues (public health, social stability, etc.), determined by the specifics of the development of neighboring territories;

- Opportunities for a rapid reversal of trends in spatial dynamics are limited by the inertial component of development (Path Dependence).

The considered provisions of the sustainable development of a local system touch upon many diverse and unsystematic issues of assessing the transformation of a heterogeneous spatial system. Most of these assessment questions are interdisciplinary, specific and far from being fully methodological. Such estimates are associated with consideration of the mathematical foundations of various forms of stability, consideration of a certain region of the phase space, return to the starting point [7]. The adaptation of the stability assessment toolkit for regional systems is inevitably associated with the integration of both the classical mathematical apparatus and interdisciplinary foundations and systems analysis.

It is advisable to consider such systems from the point of view of the interdisciplinary foundations of the development of non-equilibrium structures within the framework of the philosophy of instability. A number of important aspects of the considered temporal instability (nonlinearity of processes, heterogeneity of the considered environment, synergetic factors, etc.) directly determine the classification of objects, delimitation of zones, the formation of layers of the graphic basis for the considered neogeography models and estimates of the limits of environmental destruction. In conditions of multidirectional action of many factors and the emergence of new ones, the transformation trajectories of such systems at certain moments can be unstable. In the system-wide framework, stability is a fundamental property that characterizes the ability of systems to exist, and if a system is fundamentally unstable, then it cannot exist. Within the framework of the systemic paradigm, the national economy is always in a state of equilibrium and has many probable, normal states of the system. In such cases, a combination of several principles for assessing sustainability within the framework of a multistage research procedure is inevitable. It is also determined by intermediate estimates. The organization of such procedures involves updating the foundations of the analysis of spatial development within the framework of the methodology of soft systems - the application of systems thinking to non-systematic situations [8]. The range of possible solutions for organizing procedures is very wide here. 


\section{Results}

The article discusses the basics of research on the formation of models of sustainable development of the complex system natural forest area $\&$ urban environment. This system determines the development of the central part (core) of the agglomeration of the industrial center of the Southern Urals - Chelyabinsk (with a population of more than a million people). The key object of the natural recreational framework of the system is the Chelyabinsk urban pine forest (further - pine forest), unique in its location and geological history, 2.5 by $5-6 \mathrm{~km}$ in size, located $3 \mathrm{~km}$ from the center of the metropolis with high-rise residential buildings near the borders. Pine forest is a specially protected natural area and the main recreational object of the surrounding urban environment, which provides a high capitalization of the adjacent territories.

In the core of the Chelyabinsk agglomeration, pine forest is the only large-scale natural forest and at the same time a park. Pine forest, being a relict natural ecosystem, with a total area of about $12 \mathrm{sq}$. km, has retained some of its natural features. However, under the conditions of an ever increasing anthropogenic load, pine forest loses the stability of its natural component and has ambiguous prospects. The urban environment of one of the most ecologically problematic cities in Russia - Chelyabinsk - is distinguished by a high level of technogenic pollution. In turn, the consequences of irreversible changes associated with the recreational digression of the pine forest inevitably affect the development prospects of the megalopolis and adjacent territories.

Taking into account all of the above, visualization in transformation models of heterogeneous systems of the natural forest area \& urban environment format is associated with multi-level problem-oriented zoning and is carried out at several levels with increasing detail. The first level concerns the surrounding urban environment (characteristics of the quality of the living environment and infrastructure), the second - the territory of the pine forest.

At the first level, the factors of anthropogenic load in the surrounding urban environment are considered (changes in recreational areas, transport accessibility, population concentration in the adjacent territory, reduction of anthropogenic load due to the formation of alternative park zones, etc.).

At the second level, the identification of natural and anthropogenic objects is carried out with the establishment of their qualitative and quantitative characteristics, the establishment of the species composition of the pine forest, the natural or artificial nature of the origin of the stand, the presence of undergrowth, zones with high and low canopy density, with different moisture content, places of forest burns, etc.; identification of areas of the most valuable (significant) natural component (primary and conditionally primary areas of the natural forest area).

The digitization of the corresponding zones in accordance with the identified priority requirements of sustainable development (taking into account the nonlinearity of the main dependencies) provides the creation of a qualitatively new basis for considering the transformation scenarios for both the pine forest territory and the adjacent urban space.

In the results of the study, the use of the considered provisions and the corresponding tools is presented in the visualization of models natural forest area $\&$ urban environment format. In the long term, sustainable development means preserving the integrity and the possibility of renewing a unique natural forest area. For the adjacent territories, this ensures the capitalization of the urban environment and an increase in the quality of life of the population.

The construction of a sustainable development model for the green core (Chelyabinsk urban pine forest) in interaction with the urban environment of the Chelyabinsk agglomeration made it possible to identify and classify a number of important features of the 
spatial transformation of such a system (with a large number of influencing factors, internal heterogeneities and contradictions).

The general view of the system in the format urban environment - pine forest of the Chelyabinsk agglomeration is shown in Fig. 1. The territory of the pine forest and the zone of its half-hour transport accessibility, which largely determine the anthropogenic load, are presented on a different cartographic basis. On the basis of a photograph of the territory (Fig. 1-1), directions are shown for reducing the anthropogenic load when creating alternative recreational green zones with an exit from the territory of the city. On the basis of the scheme of residential buildings and the distribution of population density (Fig. 1-2), the main directions of the growth of anthropogenic load are shown (the population density by residential areas is marked in the figure by filling as it grows from light to dark).
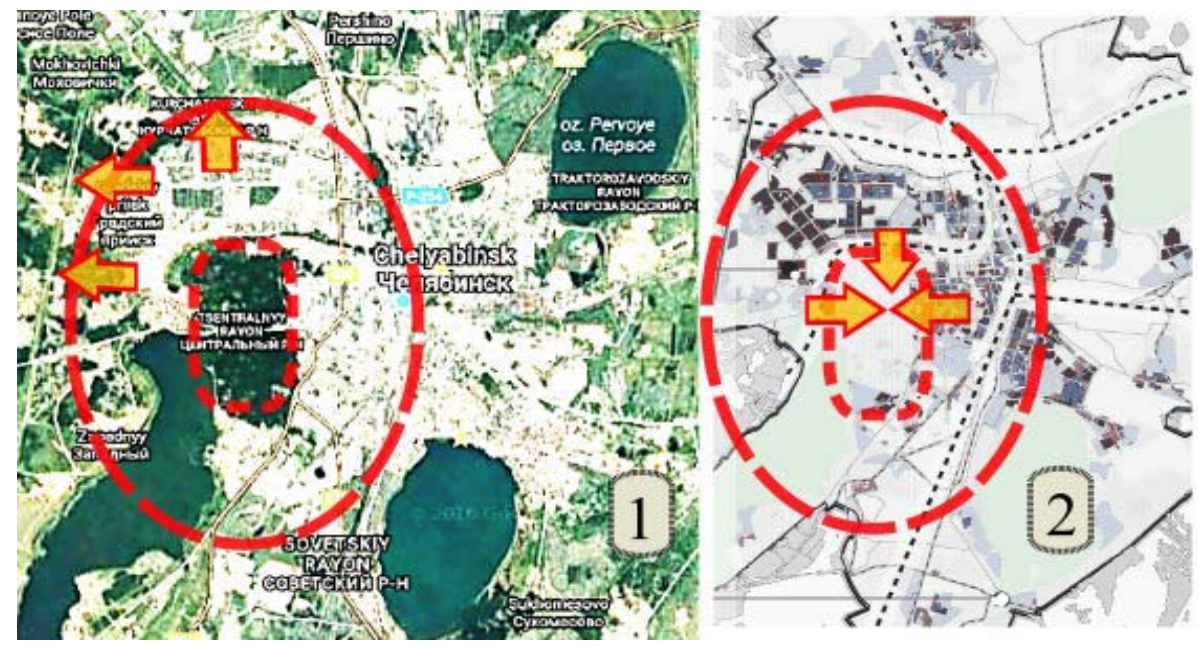

Fig. 1. General view of the system urban environment - pine forest in the Chelyabinsk agglomeration, pine forest territory and a half-hour transport accessibility zone: 1 - against the background of a photograph of the territory, 2 - against the background of the distribution scheme of residential buildings and population density.

The following features of the system in the urban environment - pine forest format have been determined. The special significance of natural pine forest for the urban environment as a spatial system-forming object is due to the shortcomings of the natural resource frame of Chelyabinsk. The provision of public green spaces in the city is several times lower than the norm. Uneven distribution of forest, forest park and park zones, represented mainly on the outskirts of the city and outside the city.

More than 600 thousand people live in the half-hour transport accessibility zone from the pine forest, which, in the absence of other noticeable recreational zones, creates the prerequisites for a multiple increase in the anthropogenic load on the pine forest (Fig. 1-2). An increase in the concentration of the population in the adjacent territories due to the mass multi-storey housing construction with a limited infrastructure determines the nonlinear growth of anthropogenic threats to the pine forest.

Additional assessment is required by dynamic factors that are rapidly changing the current situation in the urban space. Among them are the reduction of the protected buffer zone of the pine forest, construction, including residential construction, on the remaining recreational territories, etc. Separately, it should be noted the need to redistribute a part of the anthropogenic load on the external forest areas of the green ring during the transformation of the urban and suburban space of Chelyabinsk (Fig. 1-1). 
The problem of pine forest transformation is primarily determined not by internal, but by external factors of the spatial development of the urban environment in isolation from its recreational opportunities. The imbalance of the urban environment in terms of many indicators of sustainable development (starting with the presence of recreation areas and green zones) creates an uncontrolled increase in anthropogenic load with inevitable losses for the forest. Uncontrolled pressure on the pine forest in the future will inevitably lead to a decrease in the capitalization of the entire urban area with losses for the quality of life of the population.

The results of zoning of vegetation cover over the territory of the pine forest according to the photographs after processing according to a specialized author's algorithm are presented in Figure 2. Three variants of the vegetation cover of the pine forest are identified:

1 - general view of the forested area of the pine forest (various types of vegetation, including open spaces);

2 - the main areas of the forest stand, primarily of conifers (mainly scots pine);

3 - the most preserved conditionally primary and primary pine areas.

The structure of the pine forest territory is considered in the context of recent territorial transformations. There is a partial change in the boundaries of the natural forest area and an increasingly obvious fragmentation of the pine forest. According to general estimates of the current state, the share of the most valuable part of the plantations, mainly scots pine, is slightly more than $50 \%$ (Fig. 2-2). The zones of the preserved primary and conditionally primary natural pine forest are one third less (Fig. 2-3). The general preliminary estimate also indicates the development of the trend of reduction of primary natural forest pine plantations by $7-10 \%$ over the past 5 years. There are several reasons for this: the appearance of burnt areas, open spaces, border compression, expansion of the boundaries of anthropogenic objects on the territory of the natural pine forest, growth of areas of introduced plantations, replacement of pine forests with small-leaved ones, active introduction of adventive species into the composition of the stand and undergrowth. Such changes create threats of loss of the main qualitative characteristics of the natural pine forest, primarily its renewal, destruction of the structure of the natural pine forest area within 20-25 years, and further disintegration of the boron territory into separate semi-park zones.

A change in the current negative trends in pine forest evolution requires the implementation of a set of measures that will ultimately ensure a change in the trends in the inherited trajectory of development, usually considered within the framework of the Path Dependence problem [9]. In this case, the solution to this problem is complicated by the specific relationships between biological and social factors. 

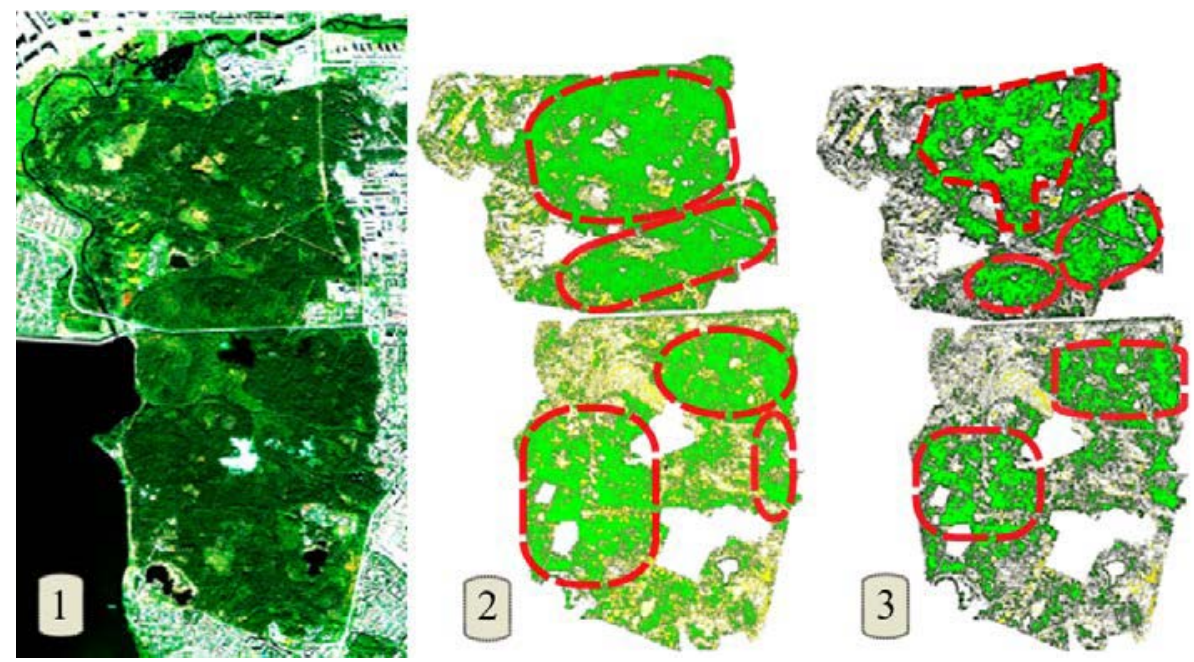

Fig. 2. Spatial distribution of vegetation over the territory of the pine forest according to photographs: 1 - general view of the forested area of the pine forest, 2 - the main areas of the forest stand, primarily of conifers (mainly scots pine), 3 - the most preserved conditionally indigenous and indigenous natural pine plots.

\section{Conclusion}

Consideration of the goals and conditions of sustainable development within the framework of multi-level zoning opens up new opportunities in solving the problems of long-term spatial development of territories, in ensuring a balance of development trajectories, prospects for synergy of new projects. Further opportunities are associated with the specification of the priorities for the development of the urban environment (primarily infrastructural) and natural objects (preservation of the natural forest area and its biological diversity).

Within the framework of neogeographic models, the cartographic base is supplemented with digital data with the possibility of expanding the boundaries of the study. Such detailing expands the possibilities of conducting deeper and more spatially targeted bio-ecological studies.

Taking into account the significant heterogeneity of the forest area and a large number of third-party objects on its territory, an alternative scenario for the development of the pine forest is associated with the allocation of dormant zones that provide better preservation of the space of the most valuable natural pine forest area, against the background of general recreational zones. The expansion of the dormant zones will further become an important element of the practice of self-restoration of the coniferous forest while ensuring a stable state of the biosphere environment.

The specificity of the development of the Chelyabinsk urban pine forest and the urban environment of the Chelyabinsk agglomeration is largely determined by the scale and severity of the contradictions. Similar problems in the development of such systems are characteristic of many industrial regions of the Urals and Siberia, as well as many other territories. The main regularities of spatial transformation considered in the study, as well as elements of the used tools, are in many respects universal and easily adaptable for heterogeneous systems of the format natural forest area \& urban environment. 


\section{Acknowledgement}

The research was funded by Russian Foundation for Basic Research, project No. 19-01000964 (in terms of research on the formation of models of sustainable development of territories).

The research was funded by Russian Foundation for Basic Research and Chelyabinsk Region, project No. 20-41-000001 (in terms of research of study of the features of the natural forest area).

\section{References}

1. B.N. Porfiryev, S.N. Bobylev, Studies on Russian Economic Development 2, 116-123 (2018) DOI: https://doi.org/10.1134/S1075700718020119

2. V.V. Akberdina, A.E.Tulin, A.A. Chursin, A.V. Iudin, The economy of the region 16, 228-241 (2020) DOI: https://doi.org/10.17059/2020-1-17

3. A. Turner, Introduction to Neogeography (Short Cuts, O'Reilly Media, 2006)

4. E. Eremchenko, S. Gorin, V. Tikunov, I. Radevski, 3-rd International Scientific Conference Geobalcanica, 121-126 (2017) DOI: http://dx.doi.org/10.18509/GBP.2017.17

5. S.S. Gordeev, S.G. Zyryanov, A.M. Sitkovskiy, ERSME-2020 217, 1-7 (2020) DOI: https://doi.org/10.1051/e3sconf/202021707021

6. S.N. Bobylev, S.V. Solovyeva, Studies on Russian Economic Development 28, 259-265, (2017) DOI: 10.1134/S1075700717030054

7. I.L. Remco, Nonlinear Dynamics 59, 173 (2010) DOI: 10.1007/s11071-009-9530-z

8. P. Checkland, Systems Research and Behavioral Science 17, 85-89 (2000) DOI: https://doi.org/10.1002/1099-1743(200011)17:1+<::AID-SRES374>3.0.CO;2-O

9. S.N. Rastvortseva, Russian journal of economic theory 15, 633-642 (2018) DOI: http://dx.doi.org/10.31063/2073-6517/2018.15-4.8 